\title{
Impact of treatment of subclinical rejection at 2 weeks after kidney transplantation, compared by analysis of 1-year histologic outcomes
}

\author{
Okjoo Lee ${ }^{1}$, Kyo Won Lee ${ }^{1}$, Jae Berm Park¹, Jung Eun Lee², Na Young Hwang ${ }^{3}$, Kyunga Kim³
}

\footnotetext{
${ }^{1}$ Division of Transplantation, Department of Surgery, Samsung Medical Center, Seoul, Korea

${ }^{2}$ Division of Nephrology, Department of Internal Medicine, Samsung Medical Center, Seoul, Korea

${ }^{3}$ Department of Biostatistics, Samsung Medical Center, Seoul, Korea
}

Background: Subclinical rejection (SCR) is associated with chronic allograft nephropathy, which is the most common cause of allograft failure in kidney transplantation (KT). Therefore, early detection and treatment of SCR through protocol biopsy can reduces the incidence of chronic allograft nephropathy and the improvement of graft survival. This study aims to evaluate the effective early detection role of routine protocol biopsy by comparing the pathologic outcome.

Methods: We retrospectively analyzed 914 KT recipients in Samsung Medical Center between August 2012 and December 2018. Of these, pediatric cases, re-transplantations and multi-organ transplantation, cyclosporine and azathioprine users, patients who were not underwent protocol biopsy, and diagnosed rejection but not treated patients were excluded. Finally, a total of 624 adult patients who were underwent protocol biopsy at post KT 2 weeks and 1 year were analyzed.

Results: After propensity score matching, patients were divided into two groups, 2-week protocol biopsy proven normal group $(n=256)$ and rejection group $(n=96)$. Before propensity matching, normal group was significant higher recipient age and ABO incompatible KT, rejection group was higher human leukocyte antigen II mismatch and proportion of deceased donor KT, the difference was corrected through matching. Rejection group showed no significant difference from normal group in the tendency of graft function (estimated glomerular filtration rate), and Kaplan-Meier curve also shown that in graft survival. In the pathologic outcomes between two groups and two periods, the pathological differences between two groups showed a decrease between two periods.

Conclusions: SCR treatment through protocol biopsy can contribute to maintenance of graft function and improvement of pathologic change.

Corresponding author: Kyo Won Lee E-mail:kw1980.lee@samsung.com

(C) The Korean Society for Transplantation

This is an Open Access article distributed under the terms of the Creative Commons Attribution Non-Commercial License (http://creativecommons.org/licenses/by-nc/4.0/) which permits unrestricted non-commercial use, distribution, and reproduction in any medium, provided the original work is properly cited. 